\title{
A pulmonary nodule in a patient with Crohn's disease
}

\author{
Akira Hokama ${ }^{1}$, Shusaku Haranaga ${ }^{2}$, Takanobu Sasaki $^{3}$, Hirofumi Matsumoto ${ }^{4}$, Tetsuya Ohira ${ }^{1}$, Atsushi Iraha ${ }^{2}$, \\ Tetsu Kinjo ${ }^{1}$, Saifun Nahar ${ }^{2}$, Jiro Fujita ${ }^{2}$ \\ Departments of ${ }^{1}$ Endoscopy, ${ }^{2}$ Infectious, Respiratory, and Digestive Medicine, ${ }^{3}$ Thoracic and Cardiovascular Surgery, and ${ }^{4}$ Pathology and \\ Oncology, Graduate School of Medicine, University of the Ryukyus, Okinawa, Japan
}

Question: A 44-year-old man presented with lower abdominal pain and diarrhea. He denied any respiratory symptoms. He had a 7-year history of ileocolonic CD. His medication included infliximab and azathioprine for 5 years, and mesalazine for 6 years. Loss of response to infliximab had been noted. Physical examination showed a body temperature of $37.2^{\circ} \mathrm{C}$ and left lower quadrant tenderness. Abdominal CT scan for bowel evaluation disclosed incidentally a nodule measuring $12 \mathrm{~mm}$ in diameter in the right lung (Fig. A, arrow). Sputum cultures and cytology showed negative results. Tests for tumor markers, $\beta$-D-glucan, the purified protein derivative, and in vitro interferon- $\gamma$ release assay were also negative. Both bronchoscopy and CT-guided percutaneous biopsy failed to diagnose the lesion. As the nodule grew and primary lung cancer was not ruled out, the lung mass was resected by video-assisted thoracoscopic surgery for definite diagnosis. Gross examination revealed a solid yellow-white nodule measuring $25 \times 20$ $\times 13 \mathrm{~mm}$ (Fig. B). What is the most likely diagnosis?
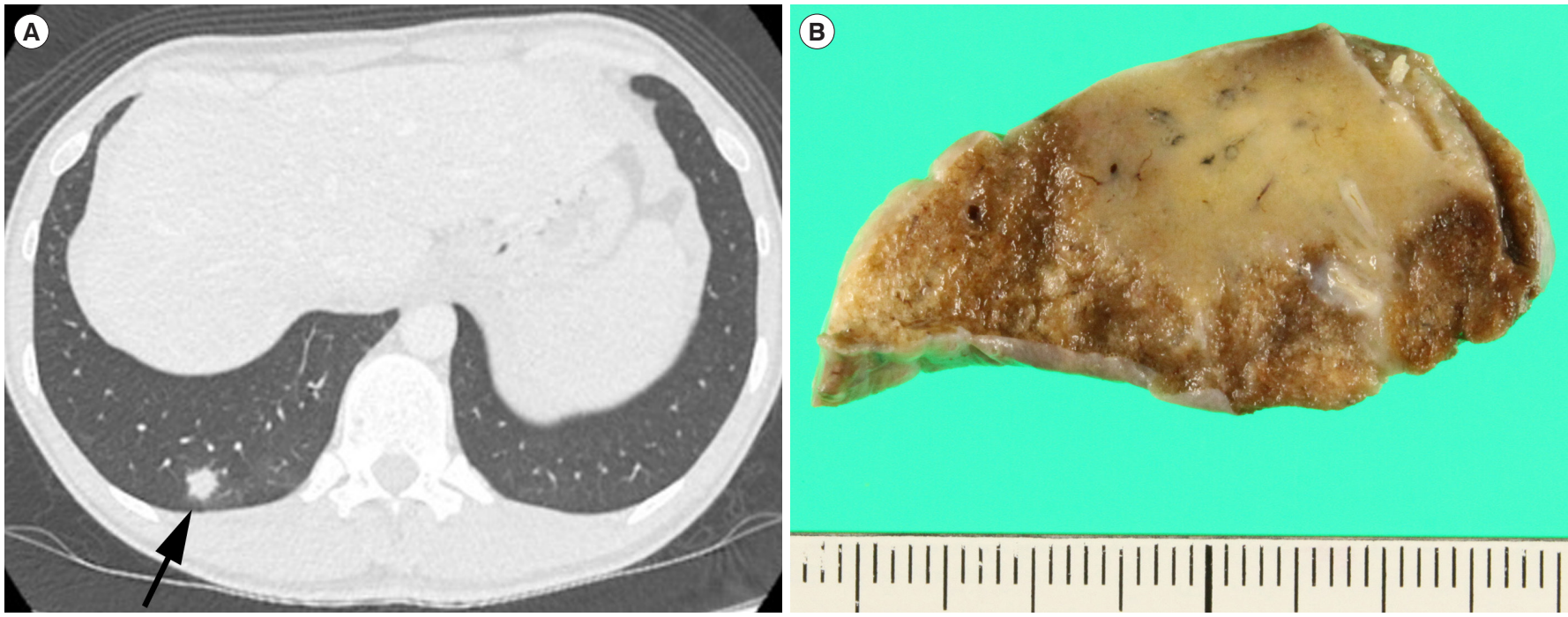

Received January 22, 2019. Accepted January 24, 2019.

Correspondence to Akira Hokama, Department of Endoscopy, Graduate School of Medicine, University of the Ryukyus, 207 Uehara, Nishihara, 0kinawa 903-0215, Japan. Tel: +81-98-895-1144, Fax: +81-98-895-1414, E-mail: hokama-a@med.u-ryukyu.ac.jp

ORCID Akira Hokama (https://orcid.org/0000-0002-8310-9989) 

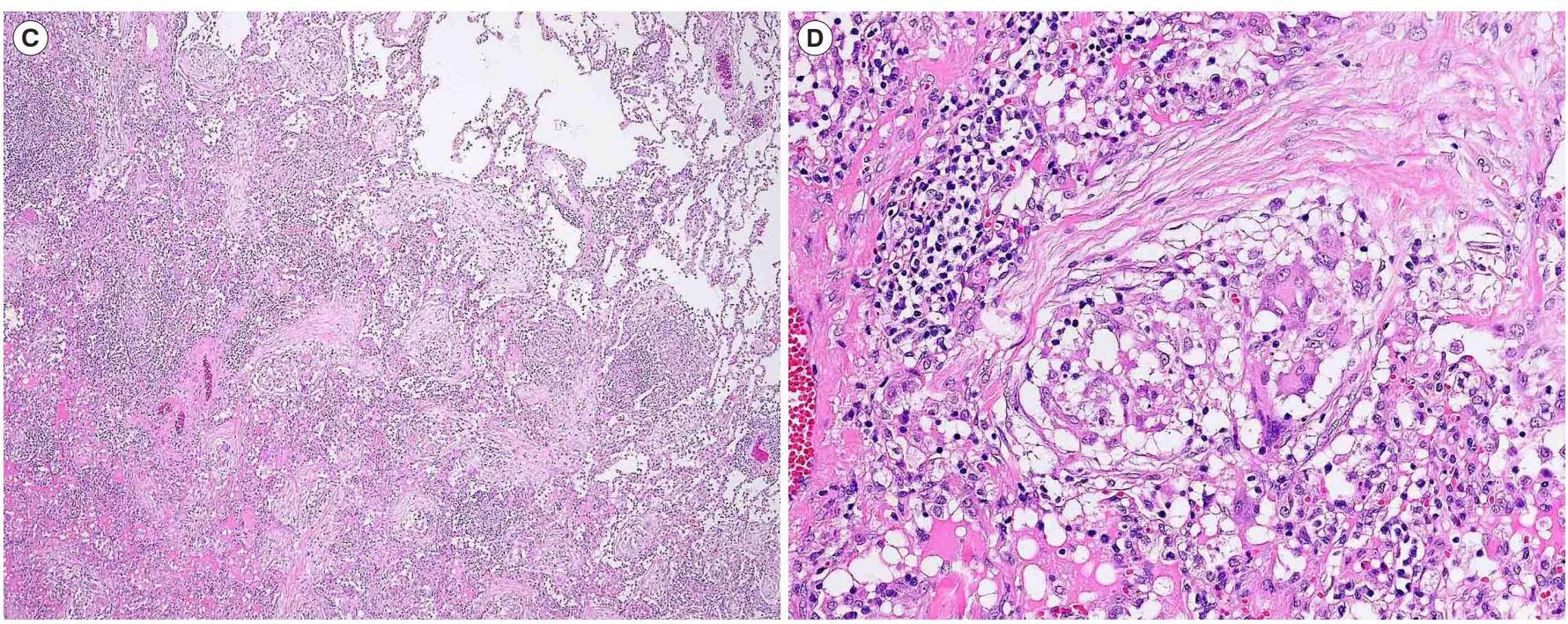

\section{Answer to the Images: Pulmonary CD with Noncaseat- ing Granulomas and Organizing Pneumonia}

The pathological examination showed numerous noncaseating granulomas and organizing pneumonia (Fig. C, H\&E, × 40). The granulomas contained typical multinucleated giant cells (Fig. D, H\&E, $\times 200$ ). Pulmonary CD with noncaseating granulomas and organizing pneumonia was diagnosed. Pulmonary lesions have not recurred during 2 years of follow-up with ustekinumab.

Bronchopulmonary involvement in $\mathrm{CD}$ is rare, but recently recognized as an important extraintestinal manifestation. These disorders consist of CD-related bronchopulmonary diseases, such as, organizing pneumonia, interstitial pneumonia, and granulomatous inflammation. ${ }^{1}$ There may be a certain link between IBD and bronchopulmonary inflammation. ${ }^{2}$ In cases of pulmonary granulomatous lesions in $\mathrm{CD}$, mycobacterial or fungal infections under immunosuppressive therapy, and sarcoidosis should be excluded, as in this case.

\section{FINANCIAL SUPPORT}

The authors received no financial support for the research, authorship, and/or publication of this article.

\section{CONFLICT OF INTEREST}

No potential conflict of interest relevant to this article was reported.

\section{AUTHOR CONTRIBUTION}

Collecting materials: Hokama A, Haranaga S, Ohira T, Iraha A, Kinjo T. Drafting the manuscript: Hokama A. Performing the surgery: Sasaki T. Examining the pathological findings: Matsumoto H. Reviewing the manuscript: Nahar S. Supervising the study: Fujita J. All authors reviewed the final version of the manuscript.

\section{REFERENCES}

1. Harbord M, Annese V, Vavricka SR, et al. The first European evidence-based consensus on extra-intestinal manifestations in inflammatory bowel disease. J Crohns Colitis 2016;10:239254.

2. Rodriguez-Roisin R, Bartolome SD, Huchon G, Krowka MJ. Inflammatory bowel diseases, chronic liver diseases and the lung. Eur Respir J 2016;47:638-650. 